\title{
Comprehensive Systematic Review to Identify putative COVID-19 Treatments: Roles for Immunomodulator and Antiviral Treatments
}

\author{
The University of Birmingham 622 COVID-19 taskforce \\ Thomas Hill $^{1}$, Mark Baker ${ }^{2}$, Lawrence Isherwood ${ }^{2}$, Lennard YW Lee Le, $^{1,3}$
}

\begin{abstract}
${ }^{1}$ Department of Oncology, Queen Elizabeth Hospital Birmingham, Mindelsohn Way, Edgbaston B15 2GW, United Kingdom ${ }^{2}$ Birmingham Medical School, College of Medical and Dental Sciences, University of Birmingham, Edgbaston, Birmingham B15 2TT ${ }^{3}$ Institute of Cancer and Genomic Sciences, University of Birmingham, Edgbaston, Birmingham B15 2TT
\end{abstract}

Corresponding Author: Lennard YW Lee, Institute of Cancer and Genomic Sciences, University of Birmingham, Edgbaston, Birmingham B15 2TT, UK. L.lee.2@bham.ac.uk, 01214143511

Conflicts of Interest: The authors declare no potential conflicts of interest.

Running Title: Immunomodulators and antivirals and putative Treatment for COVID-19

Keywords: Systematic review, COVID-19, SARS-CoV-2, treatment, trial, antiviral, immunomodulators

\begin{abstract}
Objectives: To identify putative COVID-19 treatments and identify the roles of immunomodulators and antivirals in disease management.
\end{abstract}

Design: Systematic review.

Data sources: PubMed, bioRxiv.org and medRxiv.org were searched for studies suggestive of effective treatments for COVID-19. Additional studies were identified via a snowballing method applied to the references of retrieved papers as well as a subsequent targeted search for drug names.

Review methods: Inclusion criteria included any case series or randomised control trials in any language that were published from 18th December 2019 to 18th April 2020 and described COVID-19 treatment. Of an initial 2140 studies identified from the initial search, 29 studies were found to meet the inclusion criteria and included in this comprehensive systematic review.

Results: 19 studies of antiviral treatments for COVID-19 have been reported and seven studies for immunomodulatory treatments. Six randomised controlled trials have been published with one positive trial for Hydroxychloroquine. This small study consisted of 31 patients though subsequent studies showed contradictory findings. All the remaining studies were observational studies, retrospective case reviews or non-randomised trials and these results are difficult to interpret due to methodological issues.

Conclusions: To date, an impressive number of studies have been performed in a short space of time, indicative of a resilient clinical trials infrastructure. However, there is a lack of high quality evidence to support any novel treatments for COVID-19 to be incorporated into the current standard of care. The majority of the studies of treatments for COVID-19 could only be found in pre-print servers. Future clinical reviews should therefore be Comprehensive Systematic Reviews involving pre-print studies to prevent potential unnecessary replications of clinical studies. 


\section{Introduction}

SARS-CoV-2 is a betacoronavirus of the Coronaviridae family; a group of enveloped positive single strand RNA viruses. The virus causes acute respiratory syndrome and was first identified in Hubei province, China in December 2019. Clinically, the presentation of SARS-CoV-2, COVID-19 varies from none or minor 'common cold' symptoms, to acute respiratory distress syndrome (ARDS), resulting in severely impaired respiratory function. The highly infectious nature and potential severity of the pathogenicity of COVID-19 has put significant burden on healthcare, social and economic resources. Mortality increases with age, with the highest mortality among people over 80 years of age with a case fatality rate of $21.9 \%$ (1). The standard of care treatment of COVID-19 is largely supportive, consisting of maintaining adequate oxygenation, cardiovascular perfusion and treatment of concurrent infection.

The exact mechanisms by which SARS-CoV-2 causes morbidity and mortality are poorly described. Autopsy studies have identified diffuse alveolar damage. On one hand, this might suggest a direct viral cytopathic effect on alveolar cells (2). Conversely, pneumocyte damage may also arise from ARDS, a form of rapid widespread inflammation in the lung, which arises as a consequence of activation of tissue resident macrophages, resulting in chemokine secretion leading to tissue ingress of peripheral immune cells including neutrophils and lymphocytes and further organ damage (2). There may also be a temporal dimension to the disease course of COVID-19, which may progress through different phases. It is hypothesised the initial phases and symptoms are predominantly driven by viral replication (early infection viral phase), with a late phase, and morbidity/mortality, driven by the host immune response.

The global pharmaceutical COVID-19 drug development pipeline has been developed de novo since December 2019. Broadly, two forms of treatments are under investigation. Firstly, antiviral therapy where the intention is to limit and contain viral replication, and secondly, immunomodulatory therapy with the aim to control the hyperinflammatory immune response.

In this review, we have performed a systematic review of all treatments for COVID-19 up to April $18^{\text {th }}$ 2020 and described the potential utility of antiviral and immunomodulatory strategies. This comprehensive systematic review includes all published trials, but also includes pre-print studies which are significantly more numerous and not reviewed in any previous systematic review. 
medRxiv preprint doi: https://doi.org/10.1101/2020.08.13.20174060; this version posted August 14, 2020. The copyright holder has placed this preprint (which was not certified by peer review) in the Public Domain. It is no longer restricted by copyright. Anyone can legally share, reuse, remix, or adapt this material for any purpose without crediting the original authors.

\section{Methods}

\section{Search Strategy}

A systematic review of PubMed, bioRxiv.org and medRxiv.org was performed to find original research articles providing information of interventional treatments against COVID-19. The search strategy was based on the following keywords, "COVID-19", "coronavirus", "SARS-CoV-2" and "treatment" (MeSH search terms of ((('COVID-19') OR 'Coronavirus') OR 'SARS-CoV-2') AND 'Treatment'). To expand the search, a snowballing method was applied to the references of retrieved papers with a subsequent targeted search for drug names. Research abstracts were independently reviewed by two authors to select studies that met our inclusion criteria before the full-text review of selected studies. Discrepancies and doubts of the relevance of the sources were solved by consensus with two or more authors. This systematic review was performed according to PRISMA guidelines (3).

\section{Study Selection}

Clinical studies were included in this study if they were, i) case series or RCTs, ii) from $18^{\text {th }}$ of December 2019 to $18^{\text {th }}$ of April 2020, iii) in any language and iv) describing COVID-19 treatment. Studies were excluded if, i) they reported on dietary modification including traditional herbal medications, ii) retrospective review of existing anti-hypertensives, iii) systematic reviews, iv) reporting on different forms of oxygen/ventilation, v) case reports, or vi) any commentaries or reviews.

The data and clinical findings were identified and entered into a pre-defined data extraction form, which was filled in by two reviewers. Differences were resolved by consensus. The following data were extracted; title, first author name, date of publication, publication journal, country, treatment, treatment dosage, trial design, number of patients, participant demographics, setting of treatment, outcome data, adverse events and control group. 


\section{Results}

\section{Search Results}

A search of PubMed, bioRxiv, medRxiv and a targeted search initially yielded a total of 2147 articles related to the treatment of COVID-19 (Figure 1). In total 204 articles were reviewed in detail and 29 articles were found to be relevant to this comprehensive systematic review.

In total, 13 of these reviews were published in journals, but a significant number were from a pre-print journal $(n=16,55.2 \%)$. In total, there were six RCTs, two non-randomised controlled trials, 10 observational studies, and 11 retrospective case reviews. The total number of patients exposed to these experimental drugs was 2,304 , with $56.16 \%$ being male (one study did not report gender). The majority of studies were from China $(n=21,72.4 \%)$ and Europe $(n=5,17.2 \%)$. Overall, a positive trial outcome was reported in 18 studies $(62.0 \%)$.

The clinical settings for these studies were not well defined. The majority of studies $(93.1 \%, n=27)$ investigated patients in a hospital setting. Although nine studies were conducted on patients described as severely or critically ill, only one study explicitly stated that it occurred in an intensive therapy unit (ITU) setting. There were no studies performed in an outpatient setting.

The efficacy/outcome measures were extremely heterogeneous and many studies lacked statistical analysis or significance. In terms of reported endpoints of the study, the majority were based on clinical assessment $(n=12,41.4 \%)$, some used viral markers $(n=7,24.1 \%)$ and a minority used clinical management decisions, such as decision for ITU admission or change in ventilation $(n=5,17.2 \%)$. Only two trials used survival as their end point. A significant proportion of studies did not report on adverse events $(n=8,27.5 \%)$.

There were 19 studies reporting on antiviral treatment for patients with COVID-19, seven studies investigated immunomodulatory treatment, and three studies looked at Dipyridamole, plasminogen, and low molecular weight heparin. There were six RCTs for antiviral treatments, but no RCTs for an immunomodulatory COVID-19 strategy.

\section{Antiviral Treatment}

Several different types of antiviral treatments for COVID-19 have been analysed to date, three targeting viral entry (Arbidol, Hydroxychloroquine and Chloroquine), two targeting viral polymerases (Favipiravir, Remdesivir) and three protease inhibitors (Lopinavir, Danoprevir, Ritonavir) and one of convalescent plasma.

The largest study of antiviral treatment for COVID-19 to date was for Favipiravir versus a proposed standard of care, Arbidol (4). This was a prospective randomized trial in 236 patients. This study of Favipiravir did not show any evidence of improvement of clinical recovery at day seven compared to Arbidol. It is also notable that there was also no evidence that would suggest Arbidol could be used as an antiviral treatment for COVID-19.

The anti-malarials, Chloroquine and its less toxic derivative Hydroxychloroquine are believed to have antiviral properties. This is mediated through an inhibition of terminal glycosylation of Angiotensinconverting enzyme 2 (ACE2), an enzyme attached to the outer surface (cell membranes) of cells in the lungs (5). However, it is likely that anti-inflammatory properties of these drugs also exist (6). The evidence for Chloroquine was from a $2 b$ safety study, however, this was halted early due to significant toxicities. There were two RCTs for the use of Hydroxychloroquine. The first study was performed in a total of 62 patients and found that an unusual outcome measure, 'time to clinical recovery' (TTCR), which consists of reduction of body temperature and cough, was significantly improved (7). Unfortunately, the second RCT of Hydroxychloroquine in 150 patients showed no improvement in its primary outcome - 28-day negative conversion rate (8).

Two RCTs have been performed for Lopinavir/Ritonavir; the first involved a total of 86 patients and the second 199 patients. Both studies showed no improvement in any primary or secondary outcome measures with significant toxicities (9) (10).

Finally, there is a retrospective cohort study suggestive that Remdesivir might have efficacy. Remdesivir is a nucleotide analogue prodrug which inserts into viral RNA chains, causing their premature termination (11). A retrospective case review was performed for 53 patients who were treated with Remdesivir. The majority of these patients were receiving invasive ventilation. In this cohort, $47 \%$ of patients were discharged and $13 \%$ died. However, $60 \%$ of patients had an adverse event (12). 
medRxiv preprint doi: https://doi.org/10.1101/2020.08.13.20174060; this version posted August 14, 2020. The copyright holder has placed this preprint (which was not certified by peer review) in the Public Domain. It is no longer restricted by copyright. Anyone can legally share, reuse, remix, or adapt this material for any purpose without crediting the original authors.

\section{Immunomodulatory Treatment}

Two broad immunomodulatory treatment strategies for COVID-19 have been reported. Immunomodulatory treatments were either broad in nature (corticosteroids and intravenous immunoglobulin (IVIG)) or targeted through the use of Siltuximab and Tocilizumab against IL-6 and Meplazumab against CD-147.

The largest study of immunomodulatory treatment for COVID-19 to date is for IVIG versus the standard of care (13). This was a multicentre retrospective cohort study of 325 adults with COVID-19. The study reported that its primary outcome, 28 day and 60 day mortality was not improved with IVIG treatment. However, in subgroup analysis of critical patients, IVIG seemed to reduce the 28 day mortality, reduce patient inflammatory responses and also improved some organ function.

Corticosteroids have well described systemic anti-inflammatory effects, through the up-regulation of anti-inflammatory proteins and down-regulation of pro-inflammatory proteins (14). Two studies report on the role of corticosteroids in COIVD-19 patients; both of which demonstrate corticosteroid therapy have no benefit in viral clearance, symptom resolution or mortality. The larger of the two studies, a retrospective case review of 244 patients suggested that not only did corticosteroids not improve patient's clinical outcomes, but that a higher steroid dose was significantly associated with elevated mortality risk (15). This study reported that every $10 \mathrm{mg}$ increase in Hydrocortisone equivalent dose was associated with an additional $4 \%$ mortality risk.

Finally, there were two key studies looking at the impact of targeted immunomodulatory treatment on COVID-19. Siltuximab is a monoclonal antibody that binds to and prevents the action of the proinflammatory cytokine IL-6 (16). In 21 hospitalised COVID-19 patients treated on Siltuximab, clinical improvement was observed in $33 \%$ of study participants (17). Toculizumab is a monoclonal antibody that binds to IL-6 (18). In an observational study of 30 COVID-19 patients in an intensive care setting, treatment with Toculizumab was found to reduce patient's mechanical ventilation requirements and need for ITU admission, of which $20 \%$ were discharged (19). 
medRxiv preprint doi: https://doi.org/10.1101/2020.08.13.20174060; this version posted August 14, 2020. The copyright holder has placed this preprint (which was not certified by peer review) in the Public Domain. It is no longer restricted by copyright. Anyone can legally share, reuse, remix, or adapt this material for any purpose without crediting the original authors.

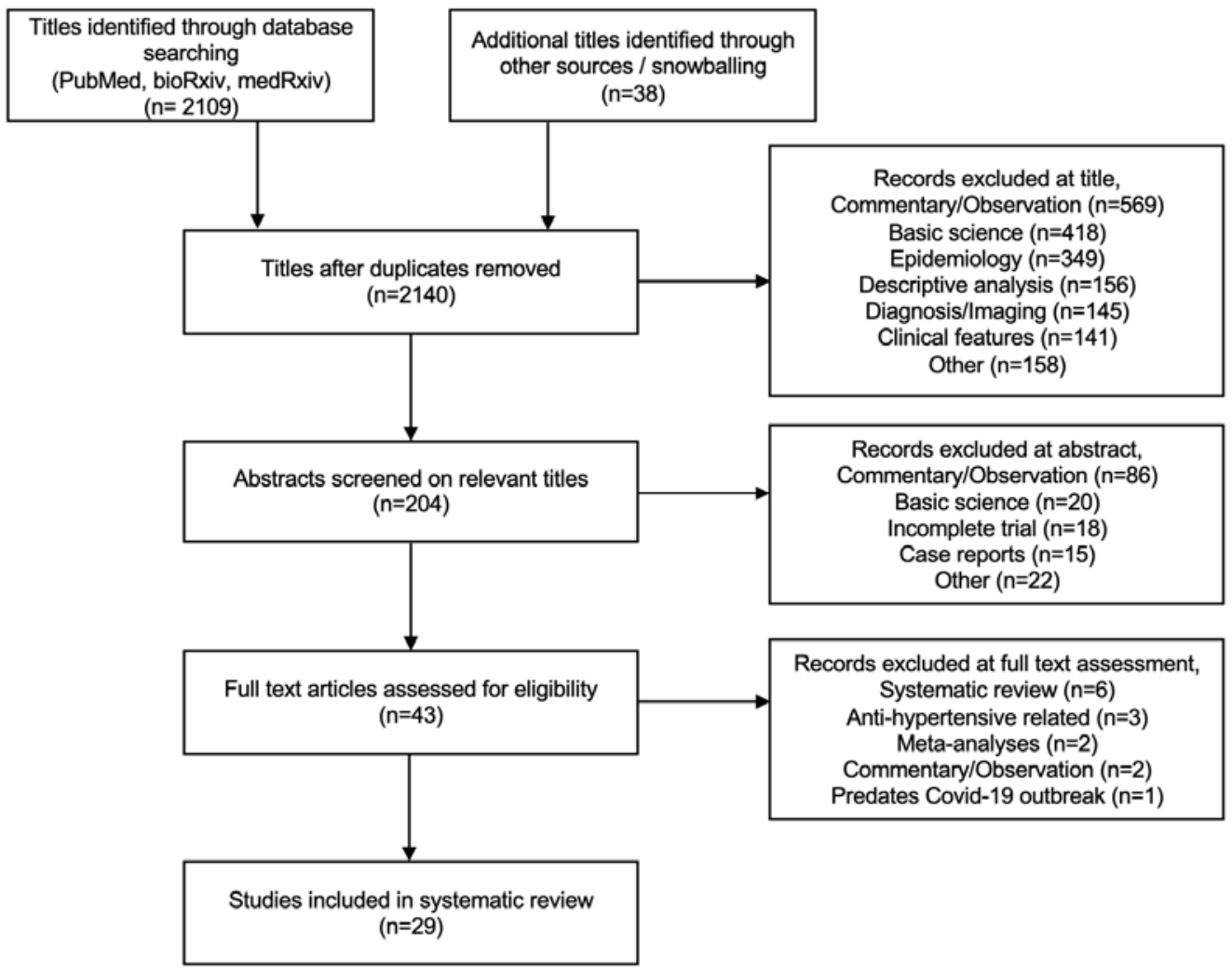

Figure 1. PRISMA flow diagram detailing comprehensive systematic review strategy 
Immunomodulatory treatment

\begin{tabular}{|c|c|c|c|c|c|c|c|c|c|c|c|c|c|}
\hline Title & Author & $\begin{array}{l}\text { Date of } \\
\text { publication }\end{array}$ & Journal & Country & Treatment & Treatment dose & Trial Design & $\begin{array}{l}\text { No. } \\
\text { patients }\end{array}$ & $\begin{array}{l}\text { Participant } \\
\text { demographics }\end{array}$ & Setting & Outcome & $\begin{array}{l}\text { Adverse } \\
\text { Events }\end{array}$ & $\begin{array}{l}\text { Control } \\
\text { Group }\end{array}$ \\
\hline $\begin{array}{l}\text { Clinical Efficacy of Intravenous } \\
\text { Immungoglobulin Therapy in Critical Patients } \\
\text { with COVIDD-19: A Multicenter Retrospective } \\
\text { Cohort Study (13) }\end{array}$ & Shao Ziyun & $11^{\text {h April }} 2020$ & medRxiv & China & $I V I G+$ Soc Vs. Soc & Varied & $\begin{array}{l}\text { Retrospective } \\
\text { case review }\end{array}$ & $\begin{array}{l}174 \text { Vs. } \\
151 \\
(n=325)\end{array}$ & $\begin{array}{l}\begin{array}{l}\text { Mean age }=58 . \\
M=189 \\
F=136\end{array} \\
\text {. }\end{array}$ & $\begin{array}{l}\text { Severe/critical } \\
\text { hospitalised patients }\end{array}$ & $\begin{array}{l}\text { No difference in mortality/discharge } \\
\text { rate. } \\
\text { Subroup showed benefit only in } \\
\text { critical COVID-19 }\end{array}$ & No information & Yes \\
\hline $\begin{array}{l}\text { Adjuvant corticosteroid therapy for critically ill } \\
\text { patients with COVID-19 (15) }\end{array}$ & Xiaofan Lu & 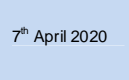 & medRxiv & China & $\begin{array}{l}\text { Corticosteroids + Soc } \\
\text { Vs. Soc }\end{array}$ & Varied & $\begin{array}{l}\text { Retrospective } \\
\text { case review }\end{array}$ & $\begin{array}{l}151 \text { vs. } \\
93 \\
(\mathrm{n}=244)\end{array}$ & $\begin{array}{l}\text { Median age }=62 . \\
M=128 \\
F=116\end{array}$ & $\begin{array}{l}\text { Critically ill TU } \\
\text { patients }\end{array}$ & Significantly elevated mortality risk & No information & Yes \\
\hline $\begin{array}{l}\text { Corticosteroid treatment of patients with } \\
\text { coronavirus disease } 2019 \text { (COVID-19) (20) }\end{array}$ & Lei Zha & 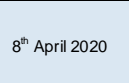 & $\begin{array}{l}\text { The Medical } \\
\text { Journal of } \\
\text { Australia }\end{array}$ & China & $\begin{array}{l}\text { Methylprednisolone + } \\
\text { Soc } \\
\text { Vs. Soc }\end{array}$ & $40 \mathrm{mg} \mathrm{OD} / \mathrm{BD}$ & $\begin{array}{l}\text { Observational } \\
\text { study }\end{array}$ & $\begin{array}{l}\begin{array}{l}11 \text { Vs. } \\
20 \\
(n=31)\end{array}\end{array}$ & $\begin{array}{l}\text { Median age }=39 . \\
M=20 \\
\mathrm{~F}=11\end{array}$ & Hospitalised patients & $\begin{array}{l}\text { No significant difference to virus } \\
\text { clearance, to discharge, or to } \\
\text { symptom resolution }\end{array}$ & No information & Yes \\
\hline $\begin{array}{l}\text { Meplazumab treats COVID-19 pneumonia: } \\
\text { an open labelled, o concurrent controlled add- } \\
\text { on cininical trial (21) }\end{array}$ & Hujije Bian & $\begin{array}{l}24^{n} \text { March } \\
2020\end{array}$ & medRxiv & China & Meplazumab Vs. Soc & $10 \mathrm{mg}$ day $1,2,5$ & Open label study & $\begin{array}{l}17 \text { vs. } \\
11 \\
(\mathrm{n}=28)\end{array}$ & $\begin{array}{l}\text { Median age= } 51 \text { (IQR } \\
49-67 \text { ) Vs. } 64 \text { (IQR } \\
43-67) \text {. } \\
\text { Genders not reported } \\
\end{array}$ & $\begin{array}{l}\text { Mild, severe \& critical } \\
\text { symptoms }\end{array}$ & $\begin{array}{l}\text { Improvement in discharge rate \& } \\
\text { severity Improvement in time to } \\
\text { negative swab }\end{array}$ & None reported & Yes \\
\hline $\begin{array}{l}\text { Use of siltuximab in patients with COVID-19 } \\
\text { pneumonia requiring ventilatory support (17) }\end{array}$ & $\begin{array}{l}\text { Giuseppe } \\
\text { Gritit }\end{array}$ & ${ }^{15 \text { April }} 2020$ & medRxiv & Ittaly & Siltuximab & $\begin{array}{l}\text { IV } 11 \mathrm{mg} / \mathrm{kg} / \mathrm{day} \\
\text { for } 1 \text { hour }\end{array}$ & $\begin{array}{l}\text { Retrospective } \\
\text { case review }\end{array}$ & $\begin{array}{l}21 \\
(\mathrm{n}=21)\end{array}$ & $\begin{array}{l}\text { Median age }=64 . \\
M=18 \\
\mathrm{~F}=3\end{array}$ & Hospitalised patients & 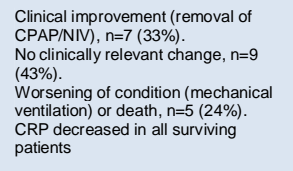 & No information & No \\
\hline $\begin{array}{l}\text { Effective Treatment of Severe CoviD-19 } \\
\text { Patients with Tocilizumab (22) }\end{array}$ & Xu Xiaoling & $\begin{array}{l}26^{6} \text { March } \\
2020\end{array}$ & Chinaxiv & China & Tocilizumab & $400 \mathrm{mg}$ & $\begin{array}{l}\text { Retrospective } \\
\text { case review }\end{array}$ & $\begin{array}{l}21 \\
(n=21)\end{array}$ & $\begin{array}{l}\text { Mean age }=56.8(16.5) \\
\begin{array}{l}M=18 \\
\mathrm{~F}=3\end{array}\end{array}$ & $\begin{array}{l}\text { Severe or critically ill } \\
\text { hospitalised patients }\end{array}$ & $\begin{array}{l}\text { Significicant clinical improvement in all, } \\
\text { dramatic reduction in temperature on } \\
\text { first day, } 19 \text { discharged. }\end{array}$ & None reported & No \\
\hline $\begin{array}{l}\text { Interleukin-6 blockade for severe COVID-19 } \\
\text { (19) }\end{array}$ & $\begin{array}{l}\text { Mathilde } \\
\text { Roumier }\end{array}$ & $\begin{array}{l}\text { Aprit } 22^{2 \pi} 2020 \\
\text { Identified atter } \\
\text { intitil searches }\end{array}$ & medRxiv & France & Tocilizumab Vs. Soc & Varied, $8 \mathrm{mg} / \mathrm{kg}$ & $\begin{array}{l}\text { Observational } \\
\text { study }\end{array}$ & $\begin{array}{l}30 \text { Vs. } \\
29 \\
(\mathrm{n}=59)\end{array}$ & $\begin{array}{l}\text { Median age }=50 . \\
M=47 \\
F=12\end{array}$ & $\begin{array}{l}\text { Severe rapidly } \\
\text { deteriorating } \\
\text { hospitalised patients }\end{array}$ & $\begin{array}{l}\text { Curbs 'cytokine storm', reduced ITU } \\
\text { admisssio, reduced mechanical } \\
\text { ventilation required }\end{array}$ & $\begin{array}{l}n=2 \text { mild } \\
\text { hepatic } \\
\text { cyloysis } \\
n=1 \text { AAP }\end{array}$ & Yes \\
\hline
\end{tabular}

Table 1: Summary of clinical trials for immunomodulatory treatments against COVID-19. 


\section{Antiviral treatment}

\begin{tabular}{|c|c|c|c|c|c|c|c|c|c|c|c|c|c|}
\hline Title & Author & $\begin{array}{l}\text { Date of } \\
\text { publication }\end{array}$ & Journal & Country & Treatment & Treatment dose & Trial design & $\begin{array}{l}\text { No. } \\
\text { patients }\end{array}$ & $\begin{array}{l}\text { Participant } \\
\text { demographics }\end{array}$ & Setting & Outcome & Adverse events & $\begin{array}{l}\text { Control } \\
\text { group }\end{array}$ \\
\hline 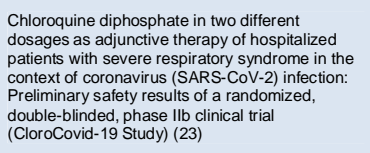 & $\begin{array}{l}\text { Mayla } \\
\text { Borba }\end{array}$ & $\begin{array}{l}{ }^{16^{\mathrm{t}} \text { April }} \\
2020\end{array}$ & medRxiv & Brazil & $\begin{array}{l}\text { Chloroquine } \\
\text { Diphosphate }\end{array}$ & $\begin{array}{l}600 \mathrm{mg} \text { BD vs. } 450 \mathrm{mg} \\
\text { BD }\end{array}$ & $\begin{array}{l}\text { Double blinded } \\
\text { randomised } \\
\text { controlled trial }\end{array}$ & $\begin{array}{l}41 \mathrm{vs} \\
40 \\
(\mathrm{n}=81)\end{array}$ & $\begin{array}{l}\text { Mean } \\
\text { age }=51.1 \\
(13.9) . \\
M=61 \\
F=20\end{array}$ & $\begin{array}{l}\text { Hospitalised } \\
\text { patients }\end{array}$ & $\begin{array}{l}\text { No significant difference, } \\
\text { recommends lower } \\
\text { dosage }\end{array}$ & $\begin{array}{l}\text { One developed } \\
\text { severe } \\
\text { rhabdomyolysis, two } \\
\text { on } 600 \mathrm{mg} \text { developed } \\
\text { ventricular fibrillation } \\
\text { before death }\end{array}$ & No \\
\hline $\begin{array}{l}\text { Hydroxychloroquine in patients with COVID-19: } \\
\text { an open-label, randomized, controlled trial (8) }\end{array}$ & Wei Tang & $\begin{array}{l}10^{\mathrm{m}} \text { April } \\
2020\end{array}$ & medRxiv & China & $\begin{array}{l}\text { Hydroxychloroquine Vs. } \\
\text { Soc }\end{array}$ & $800 \mathrm{mg}$ OD & $\begin{array}{l}\text { Randomised } \\
\text { controlled trial }\end{array}$ & $\begin{array}{l}75 \\
\text { s.75 } \\
(n=150)\end{array}$ & $\begin{array}{l}\text { Mean } \\
\text { age } 46.1 \\
(14.7) . \\
M=82 \\
\mathrm{~F}=68\end{array}$ & $\begin{array}{l}\text { Hospitalised } \\
\text { patients with CT } \\
\text { changes }\end{array}$ & $\begin{array}{l}\text { No improvement in } 28- \\
\text { day negative } \\
\text { seroconversion rate }\end{array}$ & $30 \%$ diarrhoea & Yes \\
\hline 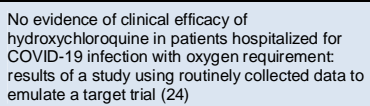 & $\begin{array}{l}\text { Matthieu } \\
\text { Mahevas }\end{array}$ & $\begin{array}{l}10^{\mathrm{th}} \text { April } \\
2020\end{array}$ & medRxiv & France & $\begin{array}{l}\text { Hydroxychloroquine Vs. } \\
\text { Soc }\end{array}$ & $600 \mathrm{mg} \mathrm{OD}$ & $\begin{array}{l}\text { Retrospective } \\
\text { case review }\end{array}$ & $\begin{array}{l}84 \mathrm{Vs} . \\
97 \\
(\mathrm{n}=181)\end{array}$ & $\begin{array}{l}\text { Median } \\
\text { age }=60 . \\
M=128 \\
\mathrm{~F}=53\end{array}$ & $\begin{array}{l}\text { Hospitalised } \\
\text { patients requiring } \\
\text { oxygen }\end{array}$ & $\begin{array}{l}\text { No difference in ITU } \\
\text { transfer rate or mortality }\end{array}$ & $\begin{array}{l}9.5 \% \text { had ECG } \\
\text { changes }\end{array}$ & Yes \\
\hline $\begin{array}{l}\text { Hydroxychloroquine and Azithromycin as a } \\
\text { treatment of COVID-19: preliminary results of an } \\
\text { open-label non-randomized clinical trial (25) }\end{array}$ & $\begin{array}{l}\text { Philippe } \\
\text { Gautret }\end{array}$ & $\begin{array}{l}22^{\mathrm{t}^{\mathrm{m}}} \text { March } \\
2020\end{array}$ & medRxiv & France & $\begin{array}{l}\text { Hydroxychloroquine + } \\
\text { Adthromycin } \\
\text { Vs. unspecified antibiotic } \\
\text { or no treatment }\end{array}$ & $\begin{array}{l}600 \mathrm{mg} \text { OD } \\
\text { All received } \\
\text { Hydroxychloroquine, } 5 \\
\text { received Azithromycin }\end{array}$ & $\begin{array}{l}\text { Open label non- } \\
\text { randomised } \\
\text { clinical trial }\end{array}$ & $\begin{array}{l}20 \mathrm{Vs} \\
16 \\
(\mathrm{n}=36)\end{array}$ & $\begin{array}{l}\text { Mean } \\
\text { age }=45.1 \\
(22.0) . \\
M=15 \\
F=21\end{array}$ & $\begin{array}{l}\text { Hospitalised } \\
\text { patients }\end{array}$ & $\begin{array}{l}\text { Significant reduction in } \\
\text { viral carriage at day } 6 . \\
\text { More efficient in } \\
\text { combined group }\end{array}$ & No information & Yes \\
\hline $\begin{array}{l}\text { Efficacy of hydroxychloroquine in patients with } \\
\text { COVID-19: results of a randomized clinical trial } \\
\text { (7) }\end{array}$ & $\begin{array}{l}\text { Zhaowei } \\
\text { Chen }\end{array}$ & $\begin{array}{l}22^{r^{d}} \text { March } \\
2020\end{array}$ & medRxiv & China & $\begin{array}{l}\text { Hydroxychloroquine Vs. } \\
\text { Soc }\end{array}$ & $400 \mathrm{mg} \mathrm{OD}$ & $\begin{array}{l}\text { Randomised } \\
\text { controlled trial }\end{array}$ & $\begin{array}{l}31 \mathrm{Vs} . \\
31 \\
(\mathrm{n}=62)\end{array}$ & $\begin{array}{l}\text { Mean } \\
\text { age }=44.7 \\
(15.3) \\
M=29 \\
F=33\end{array}$ & $\begin{array}{l}\text { Mild hospitalised } \\
\text { patients not } \\
\text { requiring oxygen }\end{array}$ & $\begin{array}{l}\text { Improved time to clinical } \\
\text { recovery }\end{array}$ & $\begin{array}{l}6.4 \% \text { rash and } \\
\text { headaches }\end{array}$ & Yes \\
\hline 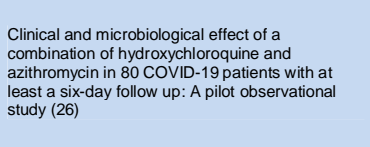 & $\begin{array}{l}\text { Philippe } \\
\text { Gautret }\end{array}$ & $\begin{array}{l}{ }_{2020}^{11^{\text {th }} \text { April }} \\
200\end{array}$ & $\begin{array}{l}\text { Travel Medicine } \\
\text { and Infectious } \\
\text { Diseases }\end{array}$ & France & $\begin{array}{l}\text { Hydroxychloroquine + } \\
\text { Azithromycin }\end{array}$ & $\begin{array}{l}200 \mathrm{mg} \\
\text { Hydroxychloroquine } \\
\text { TDS with AAththromycin } \\
500 \mathrm{mg} D 1 \text {, then 250mg } \\
\text { OD for D2-D5) } \\
\text { +Ceftriaxone if NEWS } \\
\text { score>5 }\end{array}$ & $\begin{array}{l}\text { Pilot } \\
\text { observational } \\
\text { study }\end{array}$ & $\begin{array}{l}80 \\
(n=80)\end{array}$ & $\begin{array}{l}\text { Median } \\
\text { age } 52.5 \\
(42-62.5 \\
M=43 . \\
\mathrm{F}=37\end{array}$ & $\begin{array}{l}\text { Hospitalised } \\
\text { patients }\end{array}$ & $\begin{array}{l}78(97.5 \%) \text { improved } \\
\text { clinically, rapid decrease } \\
\text { in viral load, quick ITU } \\
\text { discharge (mean } 5 \text { days) }\end{array}$ & $\begin{array}{l}\text { Nausea or vomiting } \\
2.5 \% \\
\text { Darronoea } 5 \% \\
\text { Blurred vision } 1.2 \%\end{array}$ & No \\
\hline $\begin{array}{l}\text { Clinical efficacy of lopinavirfritonaviri in the } \\
\text { treatment of Coronavirus disease } 2019 \text { (27) }\end{array}$ & X.-T.Ye & $\begin{array}{l}24^{4^{\mathrm{M}} \text { March }} \\
2020\end{array}$ & $\begin{array}{l}\text { European Review } \\
\text { for Medical and } \\
\text { Pharmacological } \\
\text { Sciences }\end{array}$ & China & $\begin{array}{l}\text { Lopinavir/Ritonavir + Soc } \\
\text { Vs. Soc }\end{array}$ & $400 / 100 \mathrm{mg}$ BD & $\begin{array}{l}\text { Observational } \\
\text { study }\end{array}$ & $\begin{array}{l}42 \text { Vs. } 5 \\
(n=47)\end{array}$ & $\begin{array}{l}\text { Range }=5-68 \\
M=22 \\
F=25\end{array}$ & $\begin{array}{l}\text { Hospitalised } \\
\text { patients }\end{array}$ & $\begin{array}{l}\text { No significant difference } \\
\text { in temperature reduction } \\
\text { except in those with } \\
\text { admission temperature } \\
>37.5 \text { degrees }\end{array}$ & None reported & Yes \\
\hline $\begin{array}{l}\text { An exploratory randomized controlled study on } \\
\text { the efficacy and safety of lopinavirritonavir or } \\
\text { arbidol treatitin adult tatients hospitilaized with } \\
\text { mild/moderate COVID-19 (ELACOI) (9) }\end{array}$ & Yueping Li & $\begin{array}{l}{ }_{2020}^{1 \mathrm{~m}^{\mathrm{m}} \text { April }} \\
20\end{array}$ & medRxiv & China & $\begin{array}{l}\text { Lopinavir/Ritonavir Vs. } \\
\text { Arbidol Vs. Soc }\end{array}$ & $\begin{array}{l}400 \mathrm{mg} / 100 \mathrm{mg} \text { BD Vs } \\
200 \mathrm{mg} \text { TDS }\end{array}$ & $\begin{array}{l}\text { Randomised } \\
\text { controlled trial }\end{array}$ & $\begin{array}{l}34 \text { Vs. } \\
35 \text { vs. } \\
17 \\
(\mathrm{n}=86)\end{array}$ & $\begin{array}{l}\text { Mean } \\
\text { ages=50.7 } \\
\text { (15.4) Vs. } \\
50.5(14.6) \text { vs. } \\
44.3 . \\
\mathrm{M}=40 \\
\mathrm{~F}=46\end{array}$ & $\begin{array}{l}\text { Mild/moderate } \\
\text { hospitalised patients }\end{array}$ & $\begin{array}{l}\text { No improvement in } \\
\text { clinical outcome }\end{array}$ & $\begin{array}{l}\text { Lopinavir/Ritonavir- } \\
35.3 \% \text {. } \\
\text { Arbidol- } 14.3 \% \\
\text { Al minor, one patient } \\
\text { with severe diarrhoea }\end{array}$ & Yes \\
\hline $\begin{array}{l}\text { A Trial of Lopinavir-Ritonaviri in Adults } \\
\text { Hospitalized with Severe Covid-19 (10) }\end{array}$ & Bin Cao & $\begin{array}{l}18^{\mathrm{m}} \text { March } \\
2020\end{array}$ & $\begin{array}{l}\text { The New England } \\
\text { journal of medicine }\end{array}$ & China & $\begin{array}{l}\text { Lopinavir/Ritonavir Vs. } \\
\text { Soc }\end{array}$ & $\begin{array}{l}400 \mathrm{mg} / \\
100 \mathrm{mg} \text { BD }\end{array}$ & $\begin{array}{l}\text { Randomised } \\
\text { controlled trial }\end{array}$ & $\begin{array}{l}99 \text { Ss. } \\
100 \\
(n=199)\end{array}$ & $\begin{array}{l}\text { Median } \\
\text { age }=58(49- \\
68) \\
M=120 \\
F=79\end{array}$ & 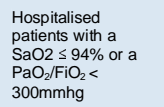 & $\begin{array}{l}\text { No difference in time to } \\
\text { clinical improvement }\end{array}$ & $\begin{array}{l}18.9 \% \text { had } \\
\text { gastrointestinal } \\
\text { adverse effects }\end{array}$ & Yes \\
\hline
\end{tabular}




\begin{tabular}{|c|c|c|c|c|c|c|c|c|c|c|c|c|c|}
\hline $\begin{array}{l}\text { Factors associated with prolonged viral shedding } \\
\text { and dimpact of Lopinavivfritonaviv treatment in } \\
\text { patients with SARS-CoV-2 infection (28) }\end{array}$ & Dan Yan & $\begin{array}{l}30^{0^{\mathrm{M}} \text { March }} \\
2020\end{array}$ & medRxiv & China & $\begin{array}{l}\text { Lopinavir/Ritonavir Vs. } \\
\text { Soc }\end{array}$ & $400 \mathrm{mg} / 100 \mathrm{mg}$ BD & $\begin{array}{l}\text { Retrospective } \\
\text { case review }\end{array}$ & $\begin{array}{l}78 \text { vs. } \\
42 \\
(\mathrm{n}=120)\end{array}$ & $\begin{array}{l}\text { Median } \\
\text { age } \\
63=52(35- \\
\text { MM) } 54 \\
F=66\end{array}$ & $\begin{array}{l}\text { Mild/severe/critical } \\
\text { hospitalised patients }\end{array}$ & $\begin{array}{l}\text { Shortened SARS-CoV-2 } \\
\text { viral shedding in } \\
\text { treatment group }\end{array}$ & No information & Yes \\
\hline $\begin{array}{l}\text { Patients of COVID-19 may benefit trom } \\
\text { sustained lopinaviri-combinened regimen and the } \\
\text { increase of eosionohil may predict the outcome } \\
\text { of COVID-19 prooression (29) }\end{array}$ & Liu Fang & $\begin{array}{l}12^{\text {th }} \text { March } \\
2020\end{array}$ & $\begin{array}{l}\text { International } \\
\text { journal of infectious } \\
\text { diseases }\end{array}$ & China & Lopinavir & $400 \mathrm{mg}$ BD & $\begin{array}{l}\text { Descriptive case } \\
\text { series }\end{array}$ & $\begin{array}{l}10 \\
(n=10)\end{array}$ & $\begin{array}{l}\text { Median } \\
\text { age }=42 \text { (IQR } \\
34-50) . \\
\text { M=4 } \\
\mathrm{F}=6\end{array}$ & Hospital inpatient & $\begin{array}{l}\text { Improvement in viral } \\
\text { load radiography, } \\
\text { hypokalaemiah, } \\
\text { lmmphopenia and } \\
\text { hypoalluminaemia }\end{array}$ & $\begin{array}{l}\text { Diarrhoea, vomiting, } \\
\text { hypokalaemia, } \\
\text { hypoalbuminaeemia }\end{array}$ & No \\
\hline $\begin{array}{l}\text { Arbidol combined with LPV/r versus LPV/ralone } \\
\text { against Corona Virus Disease 2019: A } \\
\text { retrospective cohort study (30) }\end{array}$ & Lisi Deng & $\begin{array}{l}11^{\text {th }} \text { March } \\
2020\end{array}$ & $\begin{array}{l}\text { The Journal of } \\
\text { infection }\end{array}$ & China & $\begin{array}{l}\text { Arbidol + Lopinavir / } \\
\text { Ritonavir Vs. Lopinavir / } \\
\text { Ritonavir }\end{array}$ & $\begin{array}{l}200 \mathrm{mg} \text { TDS + } \\
400 \mathrm{mg} / 100 \mathrm{mg} \text { BD Vs. } \\
400 \mathrm{mg} / 100 \mathrm{mg} \text { BD }\end{array}$ & $\begin{array}{l}\text { Retrospective } \\
\text { cohort study }\end{array}$ & $\begin{array}{l}16 \text { Vs. } \\
17 \\
(\mathrm{n}=33)\end{array}$ & $\begin{array}{l}\text { Mean } \\
\text { age }=44.6 \\
(15.73) . \\
M=17 \\
F=16\end{array}$ & $\begin{array}{l}\text { Non ITU hospital } \\
\text { inpatient }\end{array}$ & 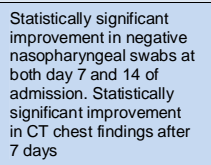 & None reported & Yes \\
\hline $\begin{array}{l}\text { Experimental Treatment with Favipiravir for } \\
\text { COVID-19: An Open-Label Control Study (31) }\end{array}$ & $\begin{array}{l}\text { Qingxian } \\
\text { Cai }\end{array}$ & $\begin{array}{l}18^{\mathrm{m}} \text { March } \\
2020\end{array}$ & Engineering & China & $\begin{array}{l}\text { Favipiravir + Interferona } \\
\text { Vs. Lopinavir / Ritonavir } \\
+ \text { + therfereron a }\end{array}$ & 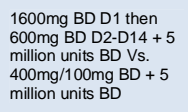 & $\begin{array}{l}\text { Open-label } \\
\text { nonrandomised } \\
\text { control study }\end{array}$ & $\begin{array}{l}35 \text { vs. } \\
45 \\
(\mathrm{n}=80)\end{array}$ & $\begin{array}{l}\text { Median } \\
\text { age }=47 \text { (IQR }= \\
\text { 35.8-61). } \\
\text { M=35 } \\
\mathrm{F}=45\end{array}$ & $\begin{array}{l}\text { Non ITU hospital } \\
\text { inpatient }\end{array}$ & 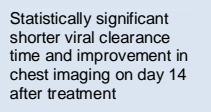 & $\begin{array}{l}\text { Less in the test arm of } \\
\text { the trial }\end{array}$ & Yes \\
\hline $\begin{array}{l}\text { First Clinical Study Using HCV Protease Inhibitor } \\
\text { Danoprevir to Treat Naive and Experienced } \\
\text { COVID-19 Patients (32) }\end{array}$ & $\begin{array}{l}\text { Hongyi } \\
\text { Chen }\end{array}$ & $\begin{array}{l}24^{\mathrm{M}} \text { March } \\
2020\end{array}$ & medRxiv & China & $\begin{array}{l}\text { Danoprevir/Ritonavir } \\
\text { A-interferon at doctors' } \\
\text { discretion }\end{array}$ & $\begin{array}{l}100 \mathrm{mg} / 100 \mathrm{mg} \\
\text { A-interferon, } 5 \text { million } \\
\text { units }\end{array}$ & $\begin{array}{l}\text { Interventional } \\
\text { clinical trial }\end{array}$ & $\underset{(n=11)}{11}$ & $\begin{array}{l}\text { Median } \\
\text { age }=44(18- \\
66)=4 \\
M=4 \\
F=7\end{array}$ & $\begin{array}{l}\text { Hospitalised } \\
\text { patients }\end{array}$ & $\begin{array}{l}\text { After } 4-11 \text { days of } \\
\text { enrolment all discharged }\end{array}$ & None reported & No \\
\hline $\begin{array}{l}\text { Faviviravir versus Arbidol for CoviD-19: A } \\
\text { Randomized Clinical Trial (4) }\end{array}$ & $\begin{array}{l}\text { Chang } \\
\text { Chen }\end{array}$ & $\begin{array}{l}{ }_{2020}^{15^{\mathrm{t}} \text { April }} \\
\end{array}$ & medRxiv & China & Favipiravir Vs. Arbidol & $\begin{array}{l}1600 \mathrm{mg} \text { twice first day } \\
\text { then } 600 \mathrm{mg} \text { BD vs. } \\
200 \mathrm{mg} \text { TDS }\end{array}$ & $\begin{array}{l}\text { Randomised } \\
\text { controlled trial }\end{array}$ & $\begin{array}{l}116 \\
\text { vs.120 } \\
(n=236)\end{array}$ & $\begin{array}{l}>65 \text { years }= \\
164 . \text { ears } \\
>655 \text { years }= \\
M=110 \\
\mathrm{M}=126\end{array}$ & $\begin{array}{l}\text { Hospitalised } \\
\text { patients with CT } \\
\text { changes }\end{array}$ & $\begin{array}{l}\text { No improvement in } \\
\text { clinical recovery rate at } \\
\text { day } 7\end{array}$ & $\begin{array}{l}\text { Raised uric acid } \\
16 / 16(16 \%) \text { in in } \\
\text { Favipiravir group }\end{array}$ & Yes \\
\hline $\begin{array}{l}\text { Compassionate Use of Remdesivir for Patients } \\
\text { with Severe Covid-19. (12) }\end{array}$ & Grein $J$ & $\begin{array}{l}{ }_{20}^{10^{\mathrm{m}} \text { April }} \\
2020\end{array}$ & $\begin{array}{l}\text { The New England } \\
\text { journal of medicine }\end{array}$ & $\begin{array}{l}\text { Various } \\
\text { United } \\
\text { States }\end{array}$ & Remdesivir & $\begin{array}{l}200 \mathrm{mg} \text { IV D1 then } \\
100 \mathrm{mg} \text { PO for D2-D10 }\end{array}$ & $\begin{array}{l}\text { Open label } \\
\text { study }\end{array}$ & $\begin{array}{l}53 \\
(n=53)\end{array}$ & $\begin{array}{l}\text { Median } \\
\text { age } 64 \text { (IQR } \\
48-71) \text {. } \\
M=40 \\
\mathrm{~F}=13\end{array}$ & $\begin{array}{l}\text { Hospitalised } \\
\text { patients with } \mathrm{SaO}_{2} \\
<94 \%\end{array}$ & $\begin{array}{l}\text { Clinical improvement } \\
\text { after } 28 \text { days in } 84 \% \text {. } \\
\text { Mortality } 13 \%\end{array}$ & $\begin{array}{l}23 \% \text { experienced } \\
\text { serious adverse } \\
\text { events axperienced } \\
60 \% \text { exper } \\
\text { some adverse event }\end{array}$ & No \\
\hline $\begin{array}{l}\text { Treatment of } 5 \text { Critically III Patients With COVID- } \\
19 \text { With Convalescent Plasma (33) }\end{array}$ & $\begin{array}{l}\text { Chenguang } \\
\text { Shen }\end{array}$ & $\begin{array}{l}27^{\mathrm{h}} \text { March } \\
2020\end{array}$ & $\begin{array}{l}\text { Journal of the } \\
\text { American medical } \\
\text { association }\end{array}$ & China & $\begin{array}{l}\text { Convalescent plasma } \\
\text { with a serum sars-cov-2- } \\
\text { specific elisa antibody } \\
\text { titre> than } 1: 100 \text { and a } \\
\text { neutralisising antibody titre } \\
>\text { than } 40\end{array}$ & $400 \mathrm{ml}$ & $\begin{array}{l}\text { Open label } \\
\text { study }\end{array}$ & $\begin{array}{l}5 \\
(n=5)\end{array}$ & $\begin{array}{l}\text { Not reported. } \\
\mathrm{M}=3 \\
\mathrm{~F}=2\end{array}$ & $\begin{array}{l}\text { Critically ill, } \\
\text { hospitalised patients }\end{array}$ & $\begin{array}{l}\text { Improvement in clinical } \\
\text { status (body } \\
\text { temperature, sofa score, } \\
\text { paoafio, virar laad, } \\
\text { serum antibody tite, } \\
\text { ARDS, ECMO support) }\end{array}$ & None reported & No \\
\hline $\begin{array}{l}\text { Effectiveness of convalescent plasma therapy in } \\
\text { severe COVID-19 patients (34) }\end{array}$ & Kai Duan & $\begin{array}{l}6^{\mathrm{n}} \text { April } \\
2020\end{array}$ & $\begin{array}{l}\text { Proceedings of the } \\
\text { National Academy } \\
\text { of Sciences of the } \\
\text { Uniten Stateates of } \\
\text { America }\end{array}$ & China & $\begin{array}{l}\text { Convalescent plasma } \\
\text { with a neutralising } \\
\text { antibody titre }>1: 640\end{array}$ & $200 \mathrm{ml}$ & $\begin{array}{l}\text { Open label } \\
\text { study }\end{array}$ & $\begin{array}{l}10 \\
(\mathrm{n}=10)\end{array}$ & $\begin{array}{l}\text { Median } \\
\text { age }=52.5 \text { (IIQR } \\
455-59.5) \\
\text { M=6. } \\
\mathrm{F}=4\end{array}$ & $\begin{array}{l}\text { Severe hospitalised } \\
\text { patients }\end{array}$ & $\begin{array}{l}\text { Significant improvement } \\
\text { in clicical symptoms, } \\
\text { oxyhacangoglobin } \\
\text { saturation and rapid } \\
\text { neutralization of viraemia }\end{array}$ & None reported & No \\
\hline $\begin{array}{l}\text { Epidemiological Features and Clinical Course of } \\
\text { Patients Infected With SARS-CoV-2 in Singapore } \\
\text { (35) }\end{array}$ & $\begin{array}{l}\text { Young, } \\
\text { Barnaby } \\
\text { Edward }\end{array}$ & $\begin{array}{l}3^{\mathrm{du}} \mathrm{March} \\
2020\end{array}$ & $\begin{array}{l}\text { Journal of the } \\
\text { American medical } \\
\text { association }\end{array}$ & Singapore & $\begin{array}{l}\text { Lopinavir / Ritonavir + } \\
\text { Soc Vs. Soc }\end{array}$ & $400 \mathrm{mg} / 100 \mathrm{mg}$ BD & $\begin{array}{l}\text { Descriptive case } \\
\text { series }\end{array}$ & $\begin{array}{l}5 \text { Vs. } 13 \\
(n=18)\end{array}$ & $\begin{array}{l}\text { Median } \\
\text { age }=47 . \\
M=9 \\
F=9\end{array}$ & $\begin{array}{l}\text { Hospital inpatient } \\
\text { including ITU }\end{array}$ & $\begin{array}{l}\text { Equivocal: } 3 / 5 \text { fever } \\
\text { resolved and oxygen } \\
\text { requirement reduced. } \\
\text { 2/5 deteriorated with } \\
\text { progressive respiratory } \\
\text { failure }\end{array}$ & $\begin{array}{l}80 \% \text { nausea, vomiting } \\
\text { and/or diarrhoea. } 60 \% \\
\text { abnormal liver } \\
\text { function tests }\end{array}$ & Yes \\
\hline
\end{tabular}

Table 2: Summary of clinical trials for antiviral treatments against COVID-19. 


\section{Other treatment}

\begin{tabular}{|c|c|c|c|c|c|c|c|c|c|c|c|c|c|}
\hline Title & Author & $\begin{array}{l}\text { Date of } \\
\text { publication }\end{array}$ & Journal & Country & Treatment & Treatment dose & Trial design & $\begin{array}{l}\text { No. } \\
\text { patients }\end{array}$ & $\begin{array}{l}\text { Participant } \\
\text { demographics }\end{array}$ & Setting & Outcome & $\begin{array}{l}\text { Adverse } \\
\text { events }\end{array}$ & $\begin{array}{l}\text { Control } \\
\text { group }\end{array}$ \\
\hline $\begin{array}{l}\text { The potential of low molecular weight heparin to } \\
\text { mitigate cytokine storm in severe COVID-19 } \\
\text { patients: a retrospective clinical study (36) }\end{array}$ & Chen Shi & $\begin{array}{l}28^{\text {th }} \text { March } \\
2020\end{array}$ & medRxiv & China & $\begin{array}{l}\text { Low molecular } \\
\text { weight heparin + } \\
\text { Soc Vs Soc }\end{array}$ & Varied & $\begin{array}{l}\text { Retrospective } \\
\text { case review }\end{array}$ & $\begin{array}{l}21 \text { vs. } \\
21 \\
(\mathrm{n}=42)\end{array}$ & $\begin{array}{l}\text { Median age }=69 . \\
M=27 \\
F=15\end{array}$ & $\begin{array}{l}\text { Hospitalised } \\
\text { patients }\end{array}$ & Improvement in LL-6 levels & $\begin{array}{l}\text { No } \\
\text { information }\end{array}$ & Yes \\
\hline $\begin{array}{l}\text { Therapeutic effects of dipyridamole on COVID-19 } \\
\text { patients with coagulation dystunction (37) }\end{array}$ & $\begin{array}{l}\text { Xiaoyan } \\
\text { Liu }\end{array}$ & $\begin{array}{l}29^{\text {th }} \\
\text { February } \\
2020\end{array}$ & medRxiv & China & $\begin{array}{l}\text { Dipyridamole Vs. } \\
\text { Soc }\end{array}$ & $150 \mathrm{mg}$ TDS & $\begin{array}{l}\text { Retrospective } \\
\text { case review }\end{array}$ & $\begin{array}{l}12 \text { Vs. } \\
10 \\
(n=22) \\
\end{array}$ & $\begin{array}{l}\text { Mean age }=53 \\
\text { V.s. } 58 . \\
\text { M=15 } \\
F=7\end{array}$ & $\begin{array}{l}\text { Mild and severe } \\
\text { cases }\end{array}$ & $\begin{array}{l}58.4 \% \text { of trial group were } \\
\text { discharged atfer } 2 \text { weeks vs. } 40 \% \\
\text { in control group }\end{array}$ & $\begin{array}{l}\text { No } \\
\text { information }\end{array}$ & Yes \\
\hline $\begin{array}{l}\text { Plasminogen improves lung lesions and hypoxemia } \\
\text { in patients with COVID-19 (38) }\end{array}$ & $\begin{array}{l}\text { Yuanyuan } \\
\text { Wu }\end{array}$ & $\begin{array}{l}10^{1 \text { th April }} \\
2020\end{array}$ & $\begin{array}{l}\text { QJM: monthly } \\
\text { journal of the } \\
\text { Association of } \\
\text { Physicians }\end{array}$ & China & $\begin{array}{l}\text { Atomization } \\
\text { inhalation of freeze- } \\
\text { dried plasminogen }\end{array}$ & $\begin{array}{l}10 \mathrm{mg} \text { in } 2 \mathrm{mg} \mathrm{saline} \mathrm{BD} \\
\text { in severelcritical, } \mathrm{OD} \text { in } \\
\text { moderate }\end{array}$ & $\begin{array}{l}\text { Observational } \\
\text { study }\end{array}$ & $\begin{array}{l}13 \\
(\mathrm{n}=13)\end{array}$ & $\begin{array}{l}\text { Median age }=48 \\
M=10 \\
\mathrm{~F}=3\end{array}$ & $\begin{array}{l}\text { Moderate, severe, } \\
\text { or critical } \\
\text { hospitalised } \\
\text { patients }\end{array}$ & $\begin{array}{l}\text { Improvement in CT, rapid } \\
\text { improvement in oxygen saturation } \\
\text { in critical, significant reduction in } \\
\text { heart rate in moderate }\end{array}$ & $\begin{array}{l}\text { None } \\
\text { reported }\end{array}$ & No \\
\hline
\end{tabular}

Table 3: Summary of clinical trials for other treatments against COVID-19. 


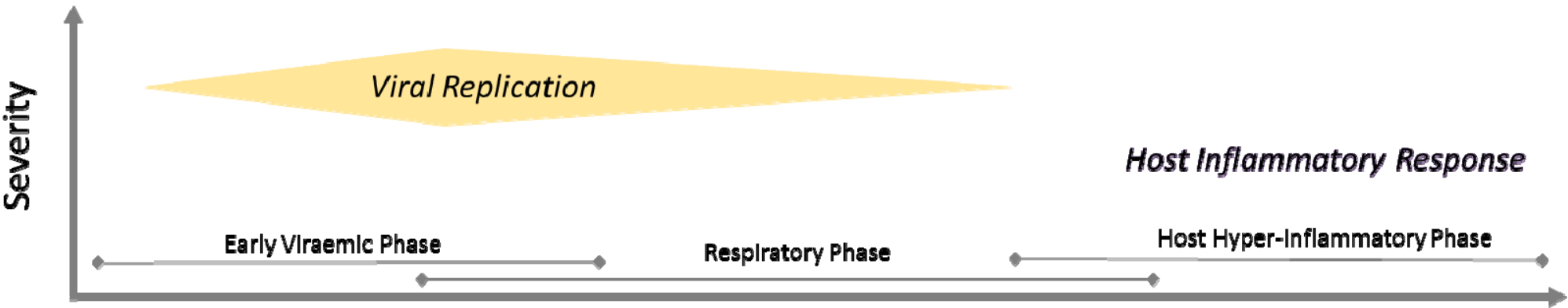

\section{Time Course}

Stages

$$
\text { Transmission Pre-Hospitalisation }
$$

Hospitalization

ITU

ARDS/SIRS/Shock/Cardiac Failure

\section{Symptoms}

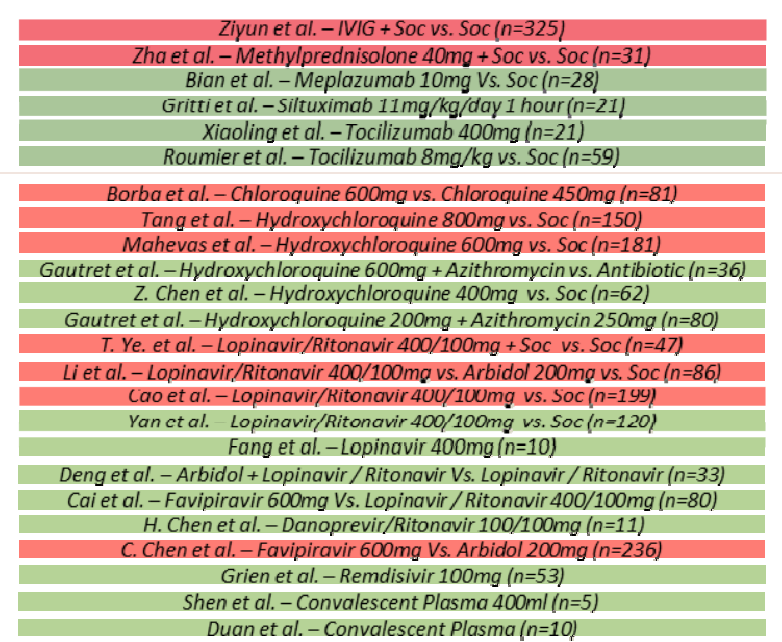

Lu et al. - Corticosteroids + Soc Vs. Soc ( $n=244)$

Ant1-Inflammatory

\section{Theraples}

AntI-VIral

Theraples

\section{Other}

Theraples

\section{Shiet al, - Low Molecular Weight Heparin + Soc vs. Soc $(n-42)$}

Wuet al. - Inhaled Freeze-Dried Plasminagen $10 \mathrm{mg}(n=13)$

Figure 2: Time course of SARS-CoV-2 infection and timing and results of clinical studies included in this Comprehensive Systematic Review

Positive Study

Negative Stue

NeutralStudy 


\section{Discussions}

The rapid global transmission of SARS-CoV-2 has caused significant strain to healthcare systems around the world and is now associated with major morbidity and mortality. The global medical research community has launched a relatively large number of clinical studies in the course of just a few months. The ability to launch these analyses in the middle of a global pandemic, for an essentially unknown disease, is a testament of the robustness of healthcare systems, excellence of individual hospitals/clinicians and is something never seen before in the history of modern medicine.

Unfortunately, this systematic review of clinical trials to date has discovered no high-quality trials identifying drug efficacy against COVID-19. One small RCT of Hydroxychloroquine/Azithromycin was positive but this has not yet been validated and had methodological flaws. However, there are many promising retrospective case studies for antivirals and immunomodulatory treatments, but these remain challenging to interpret due to self-fulfilment bias or trial design. It is also particularly surprising that there are a number of interventions which have negative/no evidence for, which are being tested in very large UK studies. This might be an artefact of a lack of recent clinical reviews or potential oversight of the evidence to date.

Nevertheless, there are a number of valuable insights which may be observed from the studies to date.

Firstly, it would appear that pre-print servers are an extremely good mechanism for disseminating literature/data. With a rapidly ensuing pandemic, the weeks/months required for publication of manuscripts in a peer-reviewed journal may mean that some studies could be needlessly replicated with potential adverse human impact. Whilst not part of the formal process for systematic reviews, the use of pre-print servers would ensure a contemporary review and facilitate a Comprehensive Systematic Review.

Secondly, most of the reported studies investigated hospitalised patients. There is only one study that has emerged out of an intensive care setting. Potentially, this may reflect the relative difficulties of performing studies or case series in ITU. Conversely. More concerningly, it is possible that drug interventions against COVID-19 in an ITU setting may have minimal effect. It might be an unsurmountable challenge for a pharmaceutical intervention to reverse respiratory and multi-organ failure, once a patient enters the hyperimmune phase with an ensuing severe cytokine release syndrome.

Finally, looking to the future, this comprehensive systematic review has identified a number of trial design strategies which would improve future clinical trials. Most of the trials treat a heterogenous patient cohort. As clinical outcomes of patients from COVID-19 vary depending on age, sex and comorbidities, a poorly defined or heterogenous trial inclusion criteria is unlikely to be compatible with efficient trial design. Furthermore, there appear to be a multitude of unvalidated surrogate study endpoints utilised for COVID-19 trials, such as ITU admissions, changes in viral load, seronegativity and cytokine panels. Survival of patients is the definitive end point and one which is rarely utilised but is of great importance. It is therefore of great regret that these trial methodological flaws continue to be repeated in newly launched nationally prioritised studies.

In summary, we have performed a Comprehensive Systematic Review of all pre-print and published articles to date to identify treatments against COVID-19. Unfortunately, there is no high-quality evidence to back any particular intervention, either antiviral or immunomodulatory to form a COVID-19 standard of care. We hope that this work will help ensure the next generation of COVID-19 clinical trials in the UK might have more efficient trial design using better targets and potentially expose less patients to risks beyond COVID-19. 


\section{Acknowledgements}

The authors thank the doctors, nurses, medical students and healthcare staff working tirelessly on the frontlines of the COVID-19 pandemic at the Queen Elizabeth Hospital Birmingham.

\section{Author Contributions}

TH, MB, LI, LL: Study design, literature review and manuscript drafting. All authors contributed equally.

Licence statement: The Corresponding Author has the right to grant on behalf of all authors and does grant on behalf of all authors, a worldwide licence to the Publishers and its licensees in perpetuity, in all forms, formats and media (whether known now or created in the future), to i) publish, reproduce, distribute, display and store the Contribution, ii) translate the Contribution into other languages, create adaptations, reprints, include within collections and create summaries, extracts and/or, abstracts of the Contribution and convert or allow conversion into any format including without limitation audio, iii) create any other derivative work(s) based in whole or part on the on the Contribution, iv) to exploit all subsidiary rights to exploit all subsidiary rights that currently exist or as may exist in the future in the Contribution, v) the inclusion of electronic links from the Contribution to third party material where-ever it may be located; and, vi) licence any third party to do any or all of the above. All research articles will be made available on an open access basis.

Patient and public involvement statement:

As we have conducted a systematic review, we have not conducted any primary research with patient involvement. We have no reason to believe that the studies we have included were not carried out with patients, carers, or members of the public, rather than on them.

Dissemination declaration: We do not plan to disseminate results to study participants and patient organisations as it is not possible and not applicable. 


\section{REFERENCES}

1. WHO. Report of the WHO-China Joint Mission on Coronavirus Disease 2019 (COVID-19) [Internet]. [cited 2020 Apr 23]. Available from: https://www.who.int/publications-detail/report-of-thewho-china-joint-mission-on-coronavirus-disease-2019-(covid-19)

2. Xu Z, Shi L, Wang Y, Zhang J, Huang L, Zhang C, et al. Pathological findings of COVID-19 associated with acute respiratory distress syndrome. Lancet Respir Med. 2020 Apr 1;8(4):420-2.

3. PRISMA. PRISMA [Internet]. TRANSPARENT REPORTING of SYSTEMATIC REVIEWS and META-ANALYSES. [cited 2020 Apr 23]. Available from: http://www.prisma-statement.org/

4. Chen C, Zhang Y, Huang J, Yin P, Cheng Z, Wu J, et al. Favipiravir versus Arbidol for COVID-19: A Randomized Clinical Trial [Internet]. Infectious Diseases (except HIV/AIDS); 2020 Mar [cited 2020 Apr 23]. Available from: http://medrxiv.org/lookup/doi/10.1101/2020.03.17.20037432

5. Hydroxychloroquine [Internet]. [cited 2020 Apr 25]. Available from: https://www.drugbank.ca/drugs/DB01611

6. Schrezenmeier E, Dörner T. Mechanisms of action of hydroxychloroquine and chloroquine: implications for rheumatology. Nat Rev Rheumatol. 2020 Mar;16(3):155-66.

7. Chen Z, Hu J, Zhang Z, Jiang S, Han S, Yan D, et al. Efficacy of hydroxychloroquine in patients with COVID-19: results of a randomized clinical trial. medRxiv. 2020 Apr 10;2020.03.22.20040758.

8. Hydroxychloroquine in patients with COVID-19: an open-label, randomized, controlled trial | medRxiv [Internet]. [cited 2020 Apr 23]. Available from: https://www.medrxiv.org/content/10.1101/2020.04.10.20060558v1

9. Li Y, Xie Z, Lin W, Cai W, Wen C, Guan Y, et al. An exploratory randomized controlled study on the efficacy and safety of lopinavir/ritonavir or arbidol treating adult patients hospitalized with mild/moderate COVID-19 (ELACOI). medRxiv. 2020 Apr 15;2020.03.19.20038984.

10. Cao B, Wang Y, Wen D, Liu W, Wang J, Fan G, et al. A Trial of Lopinavir-Ritonavir in Adults Hospitalized with Severe Covid-19. N Engl J Med [Internet]. 2020 Mar 18 [cited 2020 Apr 23]; Available from: https://www.ncbi.nlm.nih.gov/pmc/articles/PMC7121492/

11. Amirian ES, Levy JK. Current knowledge about the antivirals remdesivir (GS-5734) and GS-441524 as therapeutic options for coronaviruses. One Health. 2020 Jun 1;9:100128.

12. Grein J, Ohmagari N, Shin D, Diaz G, Asperges E, Castagna A, et al. Compassionate Use of Remdesivir for Patients with Severe Covid-19. N Engl J Med. 2020 Apr 10;NEJMoa2007016.

13. Shao Z, Feng Y, Zhong L, Xie Q, Lei M, Liu Z, et al. Clinical efficacy of intravenous immunoglobulin therapy in critical patients with COVID-19: A multicenter retrospective cohort study [Internet]. Intensive Care and Critical Care Medicine; 2020 Apr [cited 2020 Apr 23]. Available from: http://medrxiv.org/lookup/doi/10.1101/2020.04.11.20061739

14. Barnes PJ. How corticosteroids control inflammation: Quintiles Prize Lecture 2005. Br J Pharmacol. 2006 Jun;148(3):245-54.

15. Lu X, Chen T, Wang Y, Wang J, Zhang B, Li Y, et al. Adjuvant corticosteroid therapy for critically ill patients with COVID-19 [Internet]. Respiratory Medicine; 2020 Apr [cited 2020 Apr 23]. Available from: http://medrxiv.org/lookup/doi/10.1101/2020.04.07.20056390

16. Davis CC, Shah KS, Lechowicz MJ. Clinical Development of Siltuximab. Curr Oncol Rep. 2015 May 19;17(7):29.

17. Gritti G, Raimondi F, Ripamonti D, Riva I, Landi F, Alborghetti L, et al. Use of siltuximab in patients with COVID-19 pneumonia requiring ventilatory support. medRxiv. $2020 \mathrm{Apr}$ 15;2020.04.01.20048561.

18. Sebba A. Tocilizumab: The first interleukin-6-receptor inhibitor. Am J Health Syst Pharm. 2008 Aug $1 ; 65(15): 1413-8$. 
medRxiv preprint doi: https://doi.org/10.1101/2020.08.13.20174060; this version posted August 14, 2020. The copyright holder has placed this preprint (which was not certified by peer review) in the Public Domain. It is no longer restricted by copyright. Anyone can legally share, reuse, remix, or adapt this material for any purpose without crediting the original authors.

19. Roumier M, Paule R, Groh M, Vallee A, Ackermann F. Interleukin-6 blockade for severe COVID-19 [Internet]. Infectious Diseases (except HIV/AIDS); 2020 Apr [cited 2020 Apr 23]. Available from: http://medrxiv.org/lookup/doi/10.1101/2020.04.20.20061861

20. Zha L, Li S, Pan L, Tefsen B, Li Y, French N, et al. Corticosteroid treatment of patients with coronavirus disease 2019 (COVID-19). Med J Aust [Internet]. [cited 2020 Apr 23];n/a(n/a). Available from: https://onlinelibrary.wiley.com/doi/abs/10.5694/mja2.50577

21. Bian H, Zheng Z-H, Wei D, Zhang Z, Kang W-Z, Hao C-Q, et al. Meplazumab treats COVID-19 pneumonia: an open-labelled, concurrent controlled add-on clinical trial [Internet]. Infectious Diseases (except HIV/AIDS); 2020 Mar [cited 2020 Apr 23]. Available from: http://medrxiv.org/lookup/doi/10.1101/2020.03.21.20040691

22. Xu X, Han M, Li T, Sun W, Wang D, Fu B, et al. Effective Treatment of Severe COVID-19 Patients with Tocilizumab. :12.

23. Borba MGS, Val F de A, Sampaio VS, Alexandre MAA, Melo GC, Brito M, et al. Chloroquine diphosphate in two different dosages as adjunctive therapy of hospitalized patients with severe respiratory syndrome in the context of coronavirus (SARS-CoV-2) infection: Preliminary safety results of a randomized, double-blinded, phase Ilb clinical trial (CloroCovid-19 Study) [Internet]. Infectious Diseases (except HIV/AIDS); 2020 Apr [cited 2020 Apr 23]. Available from: http://medrxiv.org/lookup/doi/10.1101/2020.04.07.20056424

24. Mahevas M, Tran V-T, Roumier M, Chabrol A, Paule R, Guillaud C, et al. No evidence of clinical efficacy of hydroxychloroquine in patients hospitalized for COVID-19 infection with oxygen requirement: results of a study using routinely collected data to emulate a target trial [Internet]. Infectious Diseases (except HIV/AIDS); 2020 Apr [cited 2020 Apr 23]. Available from: http://medrxiv.org/lookup/doi/10.1101/2020.04.10.20060699

25. Gautret $\mathrm{P}$, Lagier J-C, Parola P, Hoang VT, Meddeb L, Mailhe M, et al. Hydroxychloroquine and azithromycin as a treatment of COVID-19: results of an open-label non-randomized clinical trial. Int J Antimicrob Agents. 2020 Mar;105949.

26. Gautret P, Lagier J-C, Parola P, Hoang VT, Meddeb L, Sevestre J, et al. Clinical and microbiological effect of a combination of hydroxychloroquine and azithromycin in 80 COVID-19 patients with at least a six-day follow up: A pilot observational study. Travel Med Infect Dis [Internet]. 2020 Apr 11 [cited 2020 Apr 23]; Available from: https://www.ncbi.nlm.nih.gov/pmc/articles/PMC7151271/

27. Clinical efficacy of lopinavir/ritonavir in the treatment of Coronavirus disease 2019 [Internet]. European Review. 2020 [cited 2020 Apr 23]. Available from: https://www.europeanreview.org/article/20706

28. Factors associated with prolonged viral shedding and impact of Lopinavir/Ritonavir treatment in patients with SARS-CoV-2 infection | medRxiv [Internet]. [cited 2020 Apr 23]. Available from: https://www.medrxiv.org/content/10.1101/2020.03.22.20040832v2

29. Liu F, Xu A, Zhang Y, Xuan W, Yan T, Pan K, et al. Patients of COVID-19 may benefit from sustained lopinavir-combined regimen and the increase of eosinophil may predict the outcome of COVID-19 progression. Int J Infect Dis IJID Off Publ Int Soc Infect Dis. 2020 Mar 12;

30. Deng L, Li C, Zeng Q, Liu X, Li X, Zhang H, et al. Arbidol combined with LPV/r versus LPV/r alone against Corona Virus Disease 2019: A retrospective cohort study. J Infect. 2020 Mar 11;

31. Cai Q, Yang M, Liu D, Chen J, Shu D, Xia J, et al. Experimental Treatment with Favipiravir for COVID-19: An Open-Label Control Study. Engineering [Internet]. 2020 Mar 18 [cited 2020 Apr 23]; Available from: http://www.sciencedirect.com/science/article/pii/S2095809920300631

32. Chen H, Zhang Z, Wang L, Huang Z, Gong F, Li X, et al. First Clinical Study Using HCV Protease Inhibitor Danoprevir to Treat Naive and Experienced COVID-19 Patients [Internet]. Infectious Diseases (except HIV/AIDS); 2020 Mar [cited 2020 Apr 23]. Available from: http://medrxiv.org/lookup/doi/10.1101/2020.03.22.20034041

33. Shen C, Wang Z, Zhao F, Yang Y, Li J, Yuan J, et al. Treatment of 5 Critically III Patients With COVID-19 With Convalescent Plasma. JAMA. 2020 Mar 27; 
34. Duan K, Liu B, Li C, Zhang H, Yu T, Qu J, et al. Effectiveness of convalescent plasma therapy in severe COVID-19 patients. Proc Natl Acad Sci U S A. 2020 Apr 6;

35. Young BE, Ong SWX, Kalimuddin S, Low JG, Tan SY, Loh J, et al. Epidemiologic Features and Clinical Course of Patients Infected With SARS-CoV-2 in Singapore. JAMA. 2020 Mar 3;

36. Shi $\mathrm{C}$, Wang $\mathrm{C}$, Wang $\mathrm{H}$, Yang $\mathrm{C}$, Cai $\mathrm{F}$. The potential of low molecular weight heparin to mitigate cytokine storm in severe COVID-19 patients: a retrospective clinical study | medRxiv [Internet]. [cited 2020 Apr 23]. Available from:

https://www.medrxiv.org/content/10.1101/2020.03.28.20046144v3

37. Liu X, Li Z, Liu S, Chen Z, Zhao Z, Huang Y, et al. Therapeutic effects of dipyridamole on COVID19 patients with coagulation dysfunction [Internet]. Infectious Diseases (except HIV/AIDS); 2020 Feb [cited 2020 Apr 23]. Available from: http://medrxiv.org/lookup/doi/10.1101/2020.02.27.20027557

38. Wu Y, Wang T, Guo C, Zhang D, Ge X, Huang Z, et al. Plasminogen improves lung lesions and hypoxemia in patients with COVID-19. QJM Mon J Assoc Physicians. 2020 Apr 10; 